\title{
Empirical and conceptual challenges for neurocognitive theories of language production
}

\section{Laurel Brehm \& Matthew Goldrick}

To cite this article: Laurel Brehm \& Matthew Goldrick (2016) Empirical and conceptual challenges for neurocognitive theories of language production, Language, Cognition and Neuroscience, 31:4, 504-507, DOI: $10.1080 / 23273798.2015 .1110604$

To link to this article: http://dx.doi.org/10.1080/23273798.2015.1110604

曲 Published online: 09 Nov 2015.

Submit your article to this journal




\section{Empirical and conceptual challenges for neurocognitive theories of language production}

\section{Laurel Brehm and Matthew Goldrick}

Department of Linguistics, Northwestern University, Evanston, IL, USA
Strijkers and Costa (2015) discuss three issues that challenge current theories of the neurocognitive dynamics of speech production. We focus on their latter two lines of argumentation. In the first of these, Strijkers and Costa (2015) draw on electrophysiological evidence to argue that interaction with other cognitive processes leads to flexibility in the spatio-temporal dynamics of spoken production processing. For example, production tasks varying in lexical retrieval requirements (naming or forcedchoice categorisation) yield distinct electrophysiological patterns during processing (Strijkers, Yum, Grainger, \& Holcomb, 2011). Strijkers and Costa (2015) then conclude with a discussion of challenges for discrete, staged models that assume a one-to-one relationship between cognitive and neural levels of description of the mind/brain.

In our commentary, we highlight the role of behavioural data in building a comprehensive neurocognitive theory: Performance places bounds on the degree of interactivity and flexibility in the production system. Limitations on flexibility make a straightforward interpretation of some of the neurocognitive data presented by Strijkers and Costa (2015) difficult. Furthermore, divergences between discrete cognitive representational structures (motivated by behavioural data) and the distributed nature of neural mechanisms challenge the assumption that there is a one-to-one relationship between cognitive and neural levels of description.

\section{Role of behaviour in a neurocognitive model}

If interactions with other cognitive processes lead to flexibility in the neural dynamics of language production, it is expected that behaviour should demonstrate similar interactions and flexibility. We begin by laying out data that provide reasons for optimism and reasons for scepticism with respect to these predictions.

\section{Flexibility and interactivity: reasons for optimism}

While Strijkers and Costa (2015) focus on single word production, we suspect that even stronger evidence for interaction with other cognitive processes will occur during sentence production. This is because planning a sentence is inherently resource demanding; within a brief time span, speakers must resolve competition between co-activated representations in order to correctly situate multiple items of the same lexical category within a hierarchically-ordered frame. Existing behavioural studies of sentence processing support the flexibility of production and its interaction with other cognitive processes:

- Competition and facilitation from co-activated phonological representations can be modulated by shifting task demands to emphasise or deemphasise grammatical processing (Heller \& Goldrick, 2014; Janssen \& Caramazza, 2009). This suggests flexibility in phonological processing driven by processing of sentential structure.

- Given that languages have multiple ways of formulating a given message, the particular structural choices speakers make are flexible - reflecting, in part, interactions with other cognitive processes. This can be seen in cross-linguistic variation in the degree of emphasis on grammatical, conceptual, and discourse factors in determining structural choice (Yamashita \& Chang, 2001).

- Such flexibility is found not only cross-linguistically but as a result of online interactions with other cognitive processes such as attention. For example, some events can be described by emphasising either the agent or patient. Transfer of a gift can be described as giving (emphasising the agent) or receiving (emphasising the patient). When describing such events, speakers' allocation of attention to the agent modulates the probability with which they will use the agent-emphasising form (whether endogenously 
directed or exogenously cued; Gleitman, January, Nappa, \& Trueswell, 2007).

\section{Limitations on flexibility: reasons for scepticism}

Though there is strong evidence in favour of flexibility in speech production, it is critical not to lose sight of the considerable body of evidence supporting limitations on flexibility internal to the production system. From the early analyses of speech errors (Garrett, 1975; Meringer \& Meyer, 1895), it has been clear that (i) there are errors that target entire lexical items versus those involving elements of sound structure and (ii) these error types are most strongly influenced by distinct sets of factors (semantic/syntactic vs. phonological, respectively). Such findings provided the motivation for the development of psycholinguistic theories that discretely separated lexical and phonological processing. While subsequent behavioural research has shown that such models are empirically inadequate (Vigliocco \& Hartsuiker, 2002), it has also shown that there are clear upper bounds on the degree of interactivity in the system (Goldrick, 2006).

To provide one clear example from this wide body of evidence, consider Rapp and Goldrick's (2000) case study of an individual with an acquired deficit to lexical selection processes. The distributional properties of this individual's errors support interaction between lexical selection and phonological processes. His semantically related errors (e.g. tiger $\rightarrow$ "lion") are influenced by similarity in meaning and form (exhibiting a significant mixed error effect). However, his performance also demonstrates strong limitations on interaction. In spite of a relatively high rate of error $(>25 \%)$, his lexical access difficulty never results in the production of purely phonological errors (e.g. tiger $\rightarrow$ "tire") suggesting that during lexical selection, such representations are only weakly activated.

The interpretation of such findings as supporting bounds on interactivity is bolstered by computational simulation studies. Dell and O'Seaghdha (1992) show that a system with only limited interaction ("globally modular, but locally interactive"; p. 300) can account for chronometric and speech error data. Rapp and Goldrick (2000; see also Goldrick, 2006) show that systems incorporating limited interaction can account for the full range of speech error data - whereas simulations of highly interactive, non-discrete systems fail to account for key findings. These results provide clear evidence that with respect to a large body of behavioural data, highly interactive, non-discrete production architectures are empirically inadequate.
An important challenge for future work is how to reconcile the limitations on interactivity observed within the production system with data supporting greater flexibility and interaction with other cognitive processes. Moving forward, addressing behavioural and neural data in an integrated fashion will be key to creating a true neurocognitive model of production.

\section{The mapping problem}

Next, we turn to the relationship between processing at the cognitive and neural levels. Strijkers and Costa (2015) highlight issues with current work that makes specific claims about the structure of cognitive processing and its relation to neural processing. At the cognitive level, this work assumes that processing involves sequential modules dedicated to distinct linguistic functions; additionally, these discrete cognitive modules are assumed to map in a one-to-one fashion onto the brain. Strijkers and Costa (2015) review findings incompatible with this set of assumptions, suggesting the mapping is frequently many-to-one (such that several linguistic functions are supported by a single brain region) or one-to-many (such that a single linguistic function is distributed across multiple brain regions). One solution to this incompatibility this is to maintain that there is a one-to-one mapping between cognition and neural representations, and modify the cognitive assumptions underlying current theories (as proposed in models such as cell assembly theory). However, as we discuss below, there are also serious issues in assuming a oneto-one mapping in the first place.

One general issue is that it is unclear how such a mapping can explain the cognitive and neural encoding of linguistic representational structure. The most successful accounts of the systematic structure of linguistic behaviour include symbolic, combinatorial representations. For example, the notion of "grammatical role" (e.g. subject vs. object) is critical for understanding a wide array of phenomena across languages (e.g. the order of words in sentences, variation in word form, etc.). These roles are combinatorial in the sense that they can systematically re-combine with different nouns to create different meanings (contrast "dog bites man" and "man bites dog"). Such data argue for distinct elements of cognitive representations that encode distinct aspects of linguistic structure. However, we believe it is exceedingly unlikely that there are discretely separated brain regions (or discretely separated neural processes) dedicated to instantiating these elements; more likely, an array of neural elements flexibly support the encoding of grammatical roles across distinct processing contexts. 
Due to this gap between what is required in cognition and what is likely in the brain, a one-to-one mapping between neural processing and cognition is not desirable. Instead, we need a non one-to-one mapping that is flexible, distributed, and yet preserves discrete distinctions between elements of linguistic computations. Given the enormous numbers of degrees of freedom in the problem space (the organisation of processing at the cognitive level, the organisation at the neural level, and the nature of the relationship between these levels), we see it as particularly crucial to build testable frameworks that allow us to define the space of theoretical possibilities.

The Integrated Connection-Symbolic Architecture (ICS; Smolensky \& Legendre, 2006) is one attempt to address the mapping problem. ICS allows us to formally specify how discrete cognitive representations can be instantiated as overlapping, distributed neural representations. It does so by defining an invertible transformation that precisely maps distinct cognitive representational states onto distinct neural states allowing for one-to-one and non-one-to-one correspondences between elements of processing at each level (e.g. connectionist units vs. grammatical roles). Critically, this framework allows us to examine a range of hypotheses regarding the mapping of cognitive and neural elements and to explore their contrasting predictions.

For example, Goldrick (2008) uses this framework to show that theories with identically structured cognitive representations but contrasting assumptions about the mapping of cognitive to neural structures can make different predictions regarding the distribution of speech errors. Localist neural representations (where each unit corresponds to a single cognitive element) yield a tight relationship between speech error probabilities and similarity in cognitive structure; greater cognitive similarity increases the probability that two forms will interact in an error. In contrast, distributed representations (where each connectionist unit participates in encoding multiple cognitive representations) can weaken and even reverse this pattern. This illustrates how ICS can allow for examination of a range of mapping theories, providing a springboard for future research into these issues.

\section{Conclusions}

As reviewed by Strijkers and Costa (2015) and Indefrey and Levelt (2004) before them, important strides have been made in developing a full neurocognitive model of language production. We would like to caution against ignoring the role of behavioural data in evaluating such models, as well as assuming a straightforward mapping between cognitive and neural levels of description. We believe that looking at where interactivity is not necessary and where mappings are not straightforward places valuable bounds around the challenge of building integrated models of mind and brain.

\section{Acknowledgements}

Thanks to Angela Fink and Rhonda McClain for helpful comments.

\section{Disclosure statement}

No potential conflict of interest was reported by the authors.

\section{Funding}

Supported by NSF [grant number BCS1344269]

\section{References}

Dell, G. S., \& O'Seaghdha, P. G. (1992). Stages of lexical access in language production. Cognition, 42, 287-314. doi:10.1016/ 0010-0277(92)90046-K

Garrett, M. F. (1975). The analysis of sentence production. In G. $\mathrm{H}$. Bower, The psychology of learning and motivation (Vol. 9, pp. 133-177). New York, NY: Academic Press.

Gleitman, L. R., January, D., Nappa, R., \& Trueswell, J. C. (2007). On the give and take between event apprehension and utterance formulation. Journal of Memory and Language, 57, 544-569. doi:10.1016/j.jml.2007.01.007

Goldrick, M. (2006). Limited interaction in speech production: Chronometric, speech error and neuropsychological evidence. Language and Cognitive Processes, 21, 817-855. doi:10.1080/01690960600824112

Goldrick, M. (2008). Does like attract like? Exploring the relationship between errors and representational structure in connectionist networks. Cognitive Neuropsychology, 25, 287313. doi:10.1080/02643290701417939

Heller, J. R., \& Goldrick, M. (2014). Grammatical constraints on phonological encoding in speech production. Psychonomic Bulletin and Review, 21, 1576-1582. doi:10. 3758/ s13423014-0616-3

Indefrey, P., \& Levelt, W. J. M. (2004). The spatial and temporal signatures of word production components. Cognition, 92, 101-144. doi:10.1016/j.cognition.2002.06.001

Janssen, N., \& Caramazza, A. (2009). Grammatical and phonological influences on word order. Psychological Science, 20, 1262-1268. doi:10.1111/j.1467-9280.2009.02429.x

Meringer, R., \& Meyer, K. (1895/1978). Versprechen und verlesen. Amsterdam: John Benjamins.

Rapp, B., \& Goldrick, M. (2000). Discreteness and interactivity in spoken word production. Psychological Review, 107, 460-499. doi:10.1037/0033-295X.107.3.460

Smolensky, P. \& Legendre, G. (2006). The harmonic mind: From neural computation to Optimality-Theoretic grammar. Cambridge, MA: MIT Press. 
Strijkers, K., \& Costa, A. (2015). The cortical dynamics of speaking: present shortcomings and future avenues. Manuscript submitted for publication.

Strijkers, K., Yum, Y. N., Grainger, J., \& Holcomb, P. J. (2011). Early goal-directed top-down influences in the production of speech. Frontiers in Psychology, 2, 371. doi:10.3389/fpsyg. 2011.00371
Vigliocco, G., \& Hartsuiker, R. J. (2002). The interplay of meaning, sound, and syntax in sentence production. Psychological Bulletin, 128(3), 442-472. doi:10.1037/00332909.128.3.442

Yamashita, H., \& Chang, F. (2001). "Long before short" preference in the production of a head-final language. Cognition, 81, B45-B55. doi:10.1016/S0010-0277(01)00121-4 